\title{
Promoción, Desarrollo y Persistencia de la Agricultura Familiar y sus Formas Asociativas: el caso de las asociaciones apícolas en la provincia de Chaco, Argentina (1999-2015) ${ }^{1,2}$
}

\author{
Marcos Andrés Urcola ${ }^{3}$
}

Resumen: El artículo se propone indagar sobre las formas asociativas que caracterizan a los actores identificados bajo la noción de "agricultura familiar" en el marco de los programas de desarrollo rural en la Argentina. Para ello, se interroga sobre la influencia de las diferentes estrategias grupales de intervención de los programas y sobre las posibilidades de desarrollo y persistencia de las asociaciones una vez finalizados los mismos. Adoptando un enfoque metodológico de tipo cualitativo y de estudio de caso, se analizan comparativamente las experiencias de los grupos y asociaciones apícolas promovidas en el marco de los programas Prodernea (1999-2007) y Prodear (2009-2015) en la provincia de Chaco, Argentina.

Palabras clave: agricultura familiar, asociativismo, desarrollo rural, Prodernea-Prodear.

Abstract: This paper is about the associative forms identified with family farm. The analysis is connected with rural development programs in Argentina. In this sense, we ask about the influence of the different group's strategies of intervention in those programs and the possibilities to develop and persist in these associations, after the programs period of intervention. The paper adopts a qualitative methodological strategy and it is a study's case approach related with the experiences of

1. Data de submissão: 25 de novembro de 2016. Data de aceite: 22 de outubro de 2017.

2. El artículo deriva de un Proyecto de Investigación Plurianual (PIP, 2013-2015) sobre "Políticas de desarrollo rural y asociaciones rurales en Argentina" con financiamiento del Consejo Nacional de Investigaciones Científicas y Técnicas (Conicet - Código: 11220120100344 CO). Una versión preliminar de este trabajo fue presentada y puesta en discusión en las sesiones del Grupo de Trabajo N ${ }^{\circ} 7$ sobre "Política y Desarrollo Rural" del Pre Congreso ALSRU realizado entre los días 18 y 21 de octubre de 2016 en la Universidad Nacional de Santiago del Estero (Argentina). Se agradecen los comentarios y sugerencias realizadas por los colegas participantes de aquel encuentro que permitieron mejorar el texto que se presenta a continuación.

3. Consejo Nacional de Investigaciones Científicas y Técnicas (Conicet), Instituto de Investigaciones de la Facultad de Ciencia Política y Relaciones Internacionales de la Universidad Nacional de Rosario (UNR). Rosario, Santa Fe, Argentina. E-mail: murcola@hotmail.com 
groups of honey producers and associations that have been developed in the frame of Prodernea (1999-2007) and Prodear (20092015) programs from a comparative point of view, both in the province of Chaco, Argentina.

Key-words: Family farm, associations, rural development, Prodernea-Prodear.

Clasificación JEL: Q12, Q13, Q18.

DOI: http://dx.doi.org/10.1590/1234-56781806-94790560207

\section{Introducción}

El tema del asociacionismo en el medio rural ha sido destacado históricamente como un factor que contribuye al desarrollo de los pequeños y medianos productores agropecuarios, especialmente de aquellos que tienen menor acceso o disponibilidad de recursos económicos y productivos. Esta concepción, tradicionalmente acotada a las formas jurídicas cooperativas, se ha ampliado en cuanto a sus alcances, y fortalecido teóricamente a partir de los estudios académicos sobre capital social (BOURDIEU, 1980; PUTNAM, 1993; WOOLCOCK, 1998; COLEMAN, 2000; LIN, 2001) así como también por los enfoques económicos neoinstitucionalistas (NORTH, 1995) que ponen énfasis en la importancia de las organizaciones y las instituciones para el desarrollo.

La rápida incorporación y difusión por parte de los organismos de financiamiento internacional y las agencias de desarrollo de esta concepción marcaron las estrategias de los programas de desarrollo rural en Latinoamérica en general y en la Argentina en particular durante las últimas tres décadas. Estos programas han promovido sistemáticamente metodologías de intervención grupales para brindar asistencia técnica, capacitación y financiamiento con el objeto de construir proyectos asociativos de diferente tipo que integren a productores y población rural vulnerable para mejorar sus condiciones de vida y sus capacidades de negociación y participación. El universo de grupos generados y de asociaciones creadas con el fin de recibir los beneficios de estos programas desde la década de 1990 hasta la actualidad ha sido significativo en cuanto a su número, heterogéneo respecto de su composición y grados de desarrollo y diferencial en materia de resultados. Para el año 2010, se puede estimar un número de 3.200 grupos asociativos creados en el marco de tres de los programas de desarrollo rural del Instituto Nacional de Tecnología Agropecuaria (Mnifundio, Cambio Rural y Prodaf) y alrededor de 8.700 en los programas del ex Ministerio de Agricultura Ganadería y Pesca de la Nación (MAGyP) (LATTUADA et al., 2015a).

Enmarcada en un estudio más amplio sobre las formas asociativas de la agricultura familiar en el agro argentino del nuevo milenio, en este artículo nos interesa reparar específicamente en las asociaciones promovidas en el marco de los programas de desarrollo rural, que pueden o no estar constituidas formalmente como cooperativas y que se caracterizan por integrar a ese vasto y diversificado universo de actores que integran la agricultura familiar, desempeñando un papel protagónico en la construcción social de los territorios rurales y su identidad.

A pesardesuimportanciaacadémica, la"agricultura familiar" no ha sido históricamente un sujeto o tema relevante de la agenda política del sector agropecuario argentino, sino más bien un agente residual de la misma y su proceso de legitimación política es tardío respecto de otros casos regionales como el brasileño (FLEXOR y GRISA, 2013). No obstante, entre fines del siglo pasado y principios del actual, la agricultura familiar fue creciendo en importancia como categoría de congregación política de diversos actores rurales y 
como problema de agenda de las políticas públicas de desarrollo rural en el país y la región (NOGUEIRA y URCOLA, 2013).

Si bien la discusión actual sobre la agricultura familiar reconoce sus antecedentes en las reflexiones latinoamericanas sobre el campesinado y su potencial revolucionario durante las décadas de 1960 y 1970 y sobre la eficiencia y la persistencia de la producción a pequeña escala en la dinámica capitalista de las cadenas agroindustriales durante las décadas de 1980 y parte de 1990 (SCHNEIDER, 2014), hay aspectos diferentes que dan cierta particularidad a los procesos políticos y sociales que están detrás de los debates actuales en torno a esta categoría y la acción colectiva de los actores identificados con la misma.

Partimos del supuesto de que la actual generalización del término agricultura familiar, que incluye una gran diversidad de actores (campesinos, colonos, chacareros o productores familiares capitalizados y aún a trabajadores rurales y población rural con actividades no agrarias), se ha presentado como un esfuerzo cognitivo y político por parte de científicos sociales, técnicos, funcionarios de la administración pública y organizaciones agrarias cuyo fin ha sido el de crear un nuevo marco de referencia para los gobiernos y las instituciones del ámbito local e internacional, donde se reconozca su papel estratégico en los procesos de desarrollo social y económico en los territorios rurales y su necesaria participación en la elaboración de políticas públicas.

En función de estos planteos, el interrogante que guía este trabajo refiere a cómo han influido las diferentes estrategias y enfoques de intervención de los programas de desarrollo rural para la promoción, desarrollo y persistencia de la agricultura familiar a través del fomento de su acción colectiva en distintas formas asociativas.

De este modo, el tipo de asociaciones que definimos como de la agricultura familiar en el presente trabajo corresponde a aquellas cuyos asociados reúnen las características que definen esta categoría en el marco de los programas de desarrollo rural bajo estudio. Como bien indica Craviotti (2014), los significados de un concepto están ligados a los usos que se les otorgue en diferentes ámbitos y contextos discursivos. Así, en el marco de la ejecución de un programa o una política, existe un ejercicio de mediación efectuada por técnicos, funcionarios y destinatarios que necesariamente ofrece matices a las definiciones y categorizaciones operativas formales o académicas. Si bien los significados y características del sujeto agricultor familiar han variado en el trascurso y devenir de los programas, resulta operativamente válido tomar como agricultores familiares a quienes efectivamente han sido destinatarios de los mismos, para indagar sobre sus principales problemáticas y tensiones en el marco de organizaciones e instrumentos de política específicos para el desarrollo de los territorios rurales.

\section{Objetivo y metodología}

Teniendo en cuenta estos aspectos, el objetivo del presente estudio ha sido analizar las experiencias asociativas de la agricultura familiar promovidas en la continuidad de las intervenciones de dos programas de desarrollo rural en la provincia de Chaco (Argentina) entre los años 1999 y 2015: el Programa de Desarrollo Rural del Noreste Argentino (PRODERNEA, 1999-2007) y el Programa de Desarrollo de Áreas Rurales (PRODEAR, 2009-2015).

Adoptando un enfoque metodológico de tipo cualitativo y de estudio de caso (YIN y HELAD, 1975; NEIMAN y QUARANTA, 2006), en este artículo analizamos comparativamente las experiencias de los grupos y asociaciones apícolas promovidas a través de estos dos programas, recurriendo a fuentes documentales de los mismos (véase FIDA, 2002; CEDES, 2007; CIET, 2007; FIDA, 2008; MAGyP, 2010) y a entrevistas con informantes clave.

Durante el mes de junio de 2013, se realizaron 15 entrevistas semi-estructuradas y en profundidad a representantes de los productores beneficiarios y personal técnico que habían participado del Prodernea y que seguían activos en el marco del Prodear. También se realizaron 15 entrevistas a responsables de la coordinación y ejecución de estos programas a nivel de las Unidades Provinciales de Ejecución y la Unidad Nacional de Coordinación (actual Unidad para el Cambio Rural - Ucar -, dependiente del ex-Ministerio de Agricultura Ganadería y Pesca de la Nación), que permitieron el acceso a muchas de las fuentes documentales utilizadas para este trabajo (relatorías de los talleres de cierre, evaluaciones e informes técnicos sobre beneficiarios y asociaciones). A su vez, durante 2015 y 2016, se actualizó la información de campo de 
296 Promoción, Desarrollo y Persistencia de la Agricultura Familiar y sus Formas Asociativas: el caso de las asociaciones apícolas en la provincia de Chaco, Argentina (1999-2015)

2013 a través del contactos telefónicos con informantes clave y la recopilación de documentos e Informes de Terminación del último de los programas (véase UCARPRODEAR, 2015; FIDA-PRODEAR, 2015; FIDA, 2016).

Dado que la validez de estos diseños de investigación se relaciona con la selección de los casos en función de las preguntas y objetivos propuestos, así como también de las fuentes de información de las que se dispone, ${ }^{4}$ los proyectos apícolas chaqueños desarrollados en el marco de ambos programas, nos han permitido señalar empírica y comparativamente las diferencias en materia de proceso y resultados de ambos enfoques de intervención, dando cuenta de momentos diferentes de contextualización de la problemática de la agricultura familiar, el desarrollo rural y el asociativismo como estrategia de intervención.

Para dar cuenta del caso trabajado, el presenta artículo se estructura de la siguiente manera: en el apartado de Resultados (3.) señalaremos, en primer lugar (3.1.), la importancia creciente que fue adquiriendo el asociativismo como estrategia de intervención y promoción de la agricultura familiar a partir de la influencia de la perspectiva teórica del capital social y los enfoques territoriales en los programas de desarrollo rural. En segundo lugar (3.2.), haremos referencia específica a las estrategias de intervención adoptadas por los programas seleccionados para este estudio, indicando sus diferencias y continuidades en materia de diseño y resultados desde el punto de vista asociativo. En tercer lugar (3.3.), analizaremos las experiencias apícolas desarrolladas en la continuidad de las intervenciones de los programas en la provincia de Chaco, destacando el mayor grado de formalización y crecimiento de sus asociaciones en el marco de la política provincial de apoyo a la cadena apícola y su importancia para el desarrollo organizacional de los productores familiares.

A continuación, se plantean cinco ejes de reflexión para la discusión (4.1.) en torno a las posibilidades de desarrollo y persistencia de la agricultura familiar en los territorios rurales y sus estrategias de promoción

4. Un estudio de casos se construye "a partir de un determinado, y siempre subjetivo y parcial, recorte empírico y conceptual de la realidad social, que conforma un tema y/o problema de investigación" (NEIMAN y QUARANTA, 2006, p. 218). Por estos motivos, tienden a focalizar su análisis a un número limitado de hechos y situaciones para su comprensión holística y conceptual. en el marco de las políticas públicas. Finalmente, en un último apartado (5.), se presenta la síntesis y Conclusiones generales del estudio.

\section{Resultados}

\subsection{Influencia de la perspectiva del Capital Social y el Desarrollo Territorial Rural en la promoción asociativa de la agricultura familiar}

La implementación de programas públicos de asistencia y desarrollo de pequeños y medianos productores rurales tuvo una importancia creciente en el fomento de formas asociativas de diversos tipos en el medio rural. Con escasos antecedentes previos en la historia Argentina, durante la década de 1990 se instalaron y multiplicaron numerosos programas de este tipo, dependientes de diferentes agencias estatales -Instituto Nacional de Tecnología Agropecuaria (Inta), Secretaría de Agricultura Ganadería, Pesca y Alimentos (SAGPyA)- y fuentes de financiamiento -Presupuesto Nacional, Banco Interamericano de Desarrollo (BID), Banco Mundial (BM), Fondo Internacional de Desarrollo Agrícola (FIDA)-.

Durante esta época, los programas fueron diversos en sus objetivos y estrategias, no obstante, en su amplia mayoría brindaban asistencia técnica, capacitación y financiamiento a través de estrategias grupales y asociativas con la intención de aumentar escala, reducir costos, ganar capacidad de negociación, mejorar los ingresos familiares o la seguridad alimentaria de los beneficiarios. En este sentido, la conformación de grupos de productores tenía un fin instrumental y complementariamente económico en estos programas.

Los resultados de estas acciones se manifestaron en miles de experiencias grupales en los territorios donde los programas tuvieron intervención, abarcando diversos grados de formalización y desarrollo organizacional, formas jurídicas, tipos de actividad productiva, comercial o reivindicativa, así como características socio-productivas de sus integrantes (BAUDRÓN, 1993; LATTUADA, 2000; SCHIAVONI, 2001; PROINDER, 2003; NEIMAN et al., 2006).

Esta perspectiva se mantuvo hasta la actualidad, pero la asociación de los beneficiarios pasó a ser un objetivo en sí mismo a partir del efecto que produce 
el avance de las concepciones provenientes de las corrientes neo-institucionalistas que destacaban el papel del capital social de una comunidad en desarrollo, es decir, la existencia y consolidación de numerosas asociaciones basadas en la cooperación de sus miembros y sus redes de colaboración. Esta concepción fue promovida por los organismos de financiamiento internacional e instalada con la adopción del paradigma del Desarrollo Territorial Rural (DTR) desde fines del siglo pasado y comienzos del presente.

Las nuevas corrientes económicas del denominado neo-institucionalismo sostienen la importancia de las instituciones y las organizaciones para resolver las imperfecciones del mercado en la asignación de recursos. La cultura, las normas y reglas que rigen una sociedad, el Estado y el capital social construido a partir de las formas de asociación y cooperación de sus miembros juegan un papel central en los procesos de desarrollo (NORTH, 1995).

Si bien la noción de capital social no era nueva y encontraba sus antecedentes en la obra de Bourdieu (1980), es una década después cuando se produce un tratamiento relevante de la misma en el debate académico de las ciencias sociales, a través de los trabajos de Coleman (2000) y Putnam (1993), y su adopción e instrumentación por los principales organismos de financiamiento internacional en el marco de sus propuestas de desarrollo (véase WORLD BANK, 1998).

Cabe destacar que la diversidad de autores y perspectivas que han abordado la temática del capital social han contribuido a la propagación de una tendencia ecléctica y polisémica del concepto y su aplicación en el marco de los programas de desarrollo promovidos por los organismos internacionales. Con el fin de ofrecer un concepto maleable a los diversos contextos de aplicación, los diferentes autores han pretendido unir y ordenar los estudios y enfoques sobre capital social (CUÉLLAR SAAVEDRA y BOLÍVAR ESPINOZA, 2009), cuestión que ha tendido a diluir la capacidad teórica y explicativa del mismo en favor de sus usos empíricos para la promoción de acciones de cooperación en el marco de estrategias de desarrollo.

De este modo, en un sentido amplio, el capital social puede ser interpretado como una red de relaciones sociales más o menos institucionalizadas y con cierta permanencia en el tiempo (BOURDIEU, 1980) en la que ciertos valores y normas informales comunes a los miembros de un grupo (PUTNAM, 1993) refuerzan la incorporación y práctica de actitudes de confianza y comportamientos de reciprocidad y de cooperación que, al estar enraizadas en una sociedad (LIN, 2001), contribuyen a estructurar sus relaciones sociales, posibilitando la colaboración de sus miembros para beneficio del conjunto de la comunidad (WOOLCOCK, 1998). Este conjunto de valores, normas, actitudes $\mathrm{y}$ redes entre personas y organizaciones en una comunidad es considerado un factor influyente en el desarrollo económico, el desempeño de las instituciones democráticas (PUTNAM, 1993) y, por lo tanto, relevante para diseñar programas orientados a promover la participación social y superar la pobreza.

En sintonía con esta perspectiva, la propuesta del DTR que se desarrolla hacia mediados de la década de 1990, se asienta en el supuesto de una multiplicidad de acciones de tipo económico-productivas e institucionales promovidas por actores públicos y privados con competencia sobre un territorio que cooperan y se articulan con actores de carácter regional y nacional, cuya sumatoria y sinergia confluirán en un proceso de desarrollo rural (SCHEJTMAN y RAMÍREZ, 2004).

En este sentido, otorga visibilidad y prioriza los lazos de la producción agropecuaria propiamente dicha y las actividades vinculadas (transformación, servicios y actividades no agropecuarias) que, teóricamente, generan mayores oportunidades de trabajo e ingresos en las zonas rurales. Por tanto, resulta valiosa en su rescate de la importancia de la agricultura familiar, del capital social local y regional, y de la necesidad de su fortalecimiento para cualquier iniciativa de desarrollo que se pretenda sostenible en el tiempo.

El concepto de agricultura familiar aparece en este mismo período como una noción más abarcadora respecto de la de pequeño productor, minifundista o campesino propio de los programas de la década de 1990 y que hacían hincapié en las condiciones de pobreza o escasez. Dado que el verdadero reto de esta propuesta era poner en marcha procesos de transformación que incluyeran a los pobres, a los excluidos y a los micro y pequeños empresarios rurales, la categoría de agricultura familiar se presentó como una noción que permitió representar a este abanico de actores (NOGUEIRA y URCOLA, 2013).

En el marco de esta perspectiva que presta atención a los anclajes territoriales de las prácticas y, 
especialmente, de los actores que intervienen en estas, resulta llamativo, al igual que en la perspectiva sobre capital social de Putnam, el rol residual que poseen las agencias estatales nacionales como agentes del desarrollo rural. Inicialmente, los abordajes sobre capital social y desarrollo de los territorios rurales han sido concebidos para actuar en el plano local y de las organizaciones sociales y productivas, dejando de lado la discusión sobre las políticas macroeconómicas e institucionales más generales. ${ }^{5}$

Con la recuperación de una mayor presencia e intervención del Estado nacional a nivel regional y, particularmente, en Argentina a partir de 2003, tanto en el discurso como en la práctica, comienzan a registrarse una serie de iniciativas tendientes a llevar adelante políticas sectoriales y de desarrollo rural en esta perspectiva. En 2004 la Secretaría de Agricultura, Ganadería, Pesca y Alimentación de la Nación (SAGPyA) encarga al BID y al FIDA el desarrollo de una estrategia de desarrollo rural para el país cuya coordinación se encomienda al RIMISP, reconocido por su autoridad en el desarrollo del paradigma del Desarrollo Territorial Rural. Los resultados de esa iniciativa se expresaron en una serie de documentos conceptuales y de relevamiento de experiencias en los territorios (véase SCHEJTMAN y BARSKY, 2008).

De este modo, la formación de grupos de beneficiarios inicialmente promovidos en el marco de los programas de desarrollo con la finalidad de facilitar el acceso a los servicios de crédito y asistencia técnica comienza a cambiar. Los denominados programas de "nueva generación" (LATTUADA et al., 2015b), como el PRODEAR, empiezan a poner énfasis en consolidar el desarrollo rural de la agricultura familiar otorgando un mayor protagonismo a las asociaciones de productores y de la población rural en los territorios, creando o fortaleciendo sus capacidades, con el objeto de constituir un capital social que trascienda la etapa de intervención temporal de los programas y se constituya en el sustento del desarrollo territorial. Desde la

5. Es evidente la vinculación inicial del DTR con una visión donde el Estado se retiraba de la agenda y de su rol como agente del desarrollo, característica de la etapa del ajuste estructural durante la década de 1990 (LATTUADA et al., 2015a). Del mismo modo, Ríos Cázares y Ríos Figueroa (1999) sostienen que el rol secundario que Putnam le otorga al Estado para la construcción de capital social, constituye una de las principales fallas de su modelo explicativo. perspectiva del capital social comienza a destacarse la necesidad de establecer conexiones entre las organizaciones de destinatarios de los programas con actores sociales de otros estratos $y$, fundamentalmente, con las instituciones públicas en todos sus niveles. ${ }^{6}$

La mayor centralidad otorgada a las formas asociativas de la agricultura familiar en su rol de intermediarias para la asignación de recursos entre los productores familiares y los servicios públicos provenientes del Estado y la cooperación internacional se presentó, como una alternativa económica para enfrentar las debilidades de los productores frente al mercado y dar sustentabilidad a las acciones emprendidas. También para generar cierto grado de empoderamiento que les brinde reconocimiento (visibilidad) y mayor capacidad de participación en los procesos de toma de decisión de las políticas públicas a partir de experiencias como el Foro Nacional de la Agricultura Familiar (FoNAF) y la Federación de Organizaciones Nucleadas de la Agricultura Familiar (FONAF). ${ }^{7}$ Por estos motivos se puede afirmar cierto grado de hibridismo y multifuncionalidad como rasgos comunes y constitutivos de estas experiencias asociativas, en la medida que su objetivo principal ha sido suplir tanto las necesidades materiales de sus miembros, como sus aspiraciones no monetarias de reconocimiento, inserción social y autonomía.

En el nuevo contexto, las iniciativas asociativas continuaron considerándose como una alternativa económica valiosa para los pequeños productores, pero el fortalecimiento de su organización social y política

6. A pesar del auge de los enfoques de intervención basados en esta perspectiva, siguen persistiendo controversias sobre si el capital social puede ser creado explícitamente a través de políticas públicas o es un subproducto de otras acciones y espacios institucionales.

7. En el Foro (2004) y la Federación (2011) han participado alrededor de 700 asociaciones de pequeños productores de distinto tipo (reivindicativas, no reivindicativas, económicas), entre las que se destacan organizaciones de carácter histórico como el Movimiento Agrario Misionero (MAM), el Movimiento Campesino de Santiago del Estero (Mocase) y el Movimiento Campesino de Misiones (Mocami). En un primer momento, el Foro tuvo el apoyo financiero del Fida a través de los programas Prodernea y Prodernoa (2005) y, posteriormente, de la Subsecretaría de Agricultura Familiar (2008) con la participación de los técnicos del Programa Social Agropecuario (PSA) y el Proinder que se integraron a su estructura y promovieron la conformación de la Federación en 2011. Sobre el proceso complejo de conformación de estos espacios puede consultarse, entre otros, Iurman (2016). 
comienza a ocupar un lugar tanto o más importante que los fines económicos, aunque con un alto nivel de riesgo de transitar desde un clientelismo técnico a un clientelismo político (DURSTON, 2001). ${ }^{8}$

En el marco del paradigma del DTR, la agricultura familiar emerge como categoría que permite aglutinar a una gran variedad de actores del mundo rural, mientras que el capital social permite pensar las intervenciones y la evaluación de resultados de los programas desde una perspectiva que no haga exclusivo hincapié en lo económico y la conducta individual de los agentes, fomentando la institucionalidad de las acciones colectivas para el desarrollo.

\subsection{Las estrategias de intervención y promoción de grupos y asociaciones en el marco del Prodernea y el Prodear}

Los programas que hemos seleccionado para este trabajo corresponden a las acciones destinadas al financiamiento de proyectos de desarrollo rural en el marco de convenios entre el gobierno argentino y el Fondo Internacional de Desarrollo Agrícola (Fida). ${ }^{9}$

El Programa de Desarrollo Rural del Noreste Argentino (Prodernea) inició su ejecución en la provincia de Misiones y posteriormente se incorporaron las provincias de Chaco, Formosa y Corrientes. Tuvo una reformulación en su diseño a partir del año 2003 y su actividad se prorrogó hasta junio de 2007. El mismo sólo alcanzó su plena ejecución a partir del año 2003, luego de haber sido reorientado y adecuado a las nuevas condiciones socio-económicas reinantes en las provincias de la región tras la crisis de 2001 (véase NOGUEIRA y URCOLA, 2015).

Inicialmente tomaba como población objetivo a los productores agropecuarios. No obstante, durante

8. El clientelismo es tematizado por la bibliografía especializada como uno de los efectos negativos que constituyen el "lado oscuro" del capital social. Estos, argumentan que el capital social viabiliza el clientelismo y esto no es problemático en sí, porque los implicados obtienen ganancias de él. Pero sí lo es desde el punto de vista de la políticas públicas porque distorsiona el acceso igualitario a los bienes públicos y desincentiva el control ciudadano sobre las acciones e instituciones del Estado (GARCÍA OJEDA, 2011).

9. Agencia internacional de financiamiento especializada de la Organización de Naciones Unidas (ONU) que se constituye en el año 1977 con la misión de erradicar la pobreza rural en los denominados "países en desarrollo". la misión de reorientación del programa (FIDA, 2002) se acordó clarificar y ampliar el alcance de la población objetivo, entendiéndose por éstos a todos los pobladores pobres rurales del área del proyecto y no solamente a los productores agropecuarios. De este modo, el Prodernea tomó como beneficiarias a todas las familias rurales pobres del NEA que habitaban en los predios rurales o en asentamientos de menos de 2.000 habitantes con cierta dotación de recursos productivos y capacidad de gestión con dificultades para el ingreso a los mercados.

El programa promovió metodologías de intervención grupales a través de la elaboración de proyectos asociativos de diferentes tipos. De este modo, los beneficiarios, para acceder a los créditos y recibir la asistencia técnica debían estar organizados en grupos. El informe de terminación del programa registro un total de 532 grupos de productores conformados, con 3.370 integrantes y 55 grupos de aborígenes con 5.890 integrantes (FIDA, 2008). La provincia de Chaco es la que contó con mayor porcentaje de beneficiarios participantes y proyectos financiados.

Si bien en el diseño original del programa se preveía la conformación de grupos de entre 15 o 20 integrantes, en todas las provincias predominó la conformación de grupos informales de hasta 5 integrantes. ${ }^{10}$ En las áreas rurales dispersas del proyecto fue muy difícil encontrar a más de cinco familias vecinas, ubicadas a distancias útiles para el funcionamiento en grupo y con la "afinidad" necesaria como para comprometerse con "garantías solidarias".

A esta primacía informal de los pequeños grupos de productores, debe agregarse que la mayoría de los grupos se conformaron sobre la base de lazos de proximidad y confianza (vecindad o familiares), reflejando la existencia de familias extensas en el territorio (en el 79\% de los casos, CEDES, 2007, p. 146). A pesar de esto y que la mayoría de los integrantes de los grupos presentaron condiciones productivas y de escala semejantes, es llamativo que no fueron mayoritarias las estrategias grupales de compra (bienes e insumos) y comercialización. ${ }^{11}$ La mayoría de los

10. El $56 \%$ de los grupos contaba con hasta 5 integrantes y el 29\% entre 6 y 10 integrantes. El 65\% de los mismos se constituyó de modo informal, el 13\% como cooperativa y el 10\% como asociación civil (CEDES, 2007).

11. El $30 \%$ compró todos los insumos en forma conjunta, el $25 \%$ compró parte de los insumos en forma conjunta (los 
300 - Promoción, Desarrollo y Persistencia de la Agricultura Familiar y sus Formas Asociativas: el caso de las asociaciones apícolas en la provincia de Chaco, Argentina (1999-2015)

mismos se conformaron con el fin de cumplir con los requisitos para acceder a los beneficios del programa y no lograron trascender sus acciones a la culminación del mismo o sin la presencia de un técnico de terreno que los convoque y organice.

Los aportes de estos procesos resultaron un elemento fundamental para el diseño y ejecución de los programas de "nueva generación" del Fida como el Programa de Desarrollo De Áreas Rurales (Prodear).

Con una duración prevista de 6 años, el área de influencia del Prodear lo constituyó la totalidad del territorio nacional, pero con delimitación de prioridad en las provincias del noreste argentino. ${ }^{12} \mathrm{Su}$ objetivo general consistió en desarrollar capacidades de organización que le permitan a la población rural incrementar sus ingresos, mejorar sus condiciones de vida y participar en los procesos de desarrollo de los territorios, con una perspectiva de equidad de género y con un uso sustentable de los recursos naturales (MAGyP, 2010).

Concretamente se propuso realizar actividades en respuesta a demandas de los beneficiarios, incluyendo mejoras en las organizaciones rurales productivas, en la gestión empresarial, en el desarrollo de negocios y en el saneamiento de títulos de propiedad de la tierra, tomando como población objetivo a los habitantes rurales pobres y a la población aborigen, distinguiéndose tres categorías de beneficiarios: a) pequeños productores minifundistas, campesinos $y$ familias que producen para la subsistencia y el mercado (con dificultades de acceso al crédito, al capital y a tecnología), con irregularidades en la tenencia de la tierra y un grado de organización relativamente débil; b) comunidades aborígenes constituidas por un número variable de familias asentadas en áreas rurales (mayoritariamente pertenecientes a las etnias Guaraní, Mocoví, Qom y Wichi); y c) jóvenes rurales pobres, en particular mujeres, con dificultades en el acceso

adquiridos con el crédito del programa) y el $45 \%$ no hizo compra conjunta de los mismos. El 14\% de los grupos (de mayor antigüedad y formalidad organizacional) vendió el total de su producción en forma conjunta, el $12 \%$ vendió parte de su producción en forma conjunta y el $66 \%$ vendió su producción en forma individual (CEDES, 2007, p. 127142).

12. Además de la provincias del NEA (Chaco, Corrientes y Misiones), el programa se ejecutó en las provincias de Entre Ríos, Santiago del Estero, San Juan y Mendoza. A pesar de que Formosa fue incluida en el diseño original, finalmente no ingresó en este programa. a la tierra y al mercado laboral, con alto potencial de migración hacia las ciudades (MAGyP, 2010).

En este programa, la agricultura familiar fue reivindicada como agente económico y sus organizaciones promovidas como representación social y política de sectores hasta ese momento invisibles; los gobiernos provinciales y las organizaciones en los territorios pasaron a ocupar un lugar central en la ejecución del programa y la pobreza dejó de ser concebida en términos absolutos y asistenciales para ser considerada en términos relativos y relacionales (NOGUEIRA y URCOLA, 2015).

A diferencia del programa anterior, el Prodear estableció su relación con los beneficiarios por intermedio de organizaciones preexistentes de diferente naturaleza y grado de consolidación (la falta de personería jurídica previa no fue un criterio excluyente). Las mismas debían estar integradas, al menos, por un $70 \%$ de sus miembros con las características de su grupo objetivo, pudiendo incorporar hasta un 30\% de miembros pertenecientes a otros sectores de la población rural.

De este modo, el objetivo original del Prodernea de incorporar al agricultor familiar a la banca comercial y a un mercado pago de servicios de asistencia técnica fue reemplazado en el Prodear por el objetivo de asegurar a sus beneficiarios el acceso al financiamiento y la asistencia técnica a través de sus propias organizaciones.

Para apoyar efectivamente estas actividades presentó instrumentos financieros específicos tales como: el Fondo de Apoyo a Emprendimientos (FAE), con recursos no reembolsables destinados al apoyo de emprendimientos socio-productivos y comerciales para acceder a nuevos mercados; el Fondo de Capitalización de Organizaciones (Foco), para capitalizar organizaciones que desarrollen sistemas de micro-financiamiento rotatorios generados por ellas mismas; y el Fondo de Inversiones (FI) para proporcionar crédito de mediano y largo plazo a inversiones de uso colectivo en proyectos aprobados en el marco de planes de negocios de las organizaciones. A través de los mismos se buscaba consolidar organizaciones con fines económicos sostenibles, creando espacios de articulación y coordinación con las instituciones de desarrollo local y otras organizaciones para ampliar el capital social (MAGyP, 2010).

Es pertinente señalar que, con esta misma lógica y en el mismo período (2011), se crea en el plano gremial 
la ya mencionada Federación de Organizaciones Nucleadas de la Agricultura Familiar (FONAF). Desde la Subsecretaría de Agricultura Familiar se fomentó la formalización jurídica de las organizaciones que participaban del Foro Nacional de la Agricultura Familiar con el fin de avanzar en la gestión de recursos sin la intermediación de los programas de desarrollo rural y apostando a una mayor autonomía respecto de los técnicos y funcionarios de la gestión de los mismos (IURMAN, 2016), aunque con los ya mencionados riesgos de pasar de un clientelismo técnico a uno político (DURSTON, 2001).

El informe de supervisión final del Prodear (FIDA, 2016), registra un total de 631 grupos que recibieron fondos (FAE) para mejorar la producción agropecuaria intrapredial y colectiva y el almacenamiento o procesamiento de productos para la comercialización y agregado de valor. A su vez, 41 cooperativas o asociaciones civiles recibieron fondos (Foco) para la capitalización de sus organizaciones (16 de ellas corresponden a la provincia de Chaco y 10 son apícolas). El menor número de organizaciones que recibió esta línea de financiamiento (FOCO) se explica por el mayor grado de consolidación asociativa que se requiere para emprender de un modo aceptable la administración de estos fondos rotatorios de micro-financiamiento. En este sentido, es importante destacar que, a pesar de las intenciones de promoción de autonomía y autogestión de las organizaciones con instrumentos como los Foco, la mayoría de los grupos se vincularon con el programa a partir de los fondos de mejora productiva similares a los del Prodernea.

Teniendo en cuenta estos aspectos, es relevante destacar que la mera existencia de grupos y asociaciones es insuficiente como indicador de procesos de desarrollo en los territorios si no existen ciertas condiciones complementarias vinculadas a las características sobre las que se fundan las asociaciones (objetivos y acciones que efectivamente llevan adelante) y las acciones del Estado como interlocutor y mediador de los procesos de inserción comercial de sus productos y representación de sus intereses. Las experiencias de los programas de desarrollo demuestran que las condiciones para que las asociaciones de la agricultura familiar se consoliden con algún grado de autonomía, no están dadas ni son extendidas sino que, por el contrario, son escasas y requieren ser construidas (LATTUADA et al., 2015a). Por ello, la posibilidad de identificarlas requiere del análisis de experiencias concretas en contextos determinados, como el caso de las organizaciones apícolas de la provincia de Chaco que les proponemos a continuación.

\subsection{Los proyectos apícolas en la provincia de Chaco}

La cadena de valor apícola cuenta con la particularidad de ofrecer una escasa complejidad. La miel sufre una exigua transformación física y son pocos los agentes queintervienen en la etapa de procesamiento. Dicha cadena se organiza en torno a cinco funciones básicas: la provisión de insumos, la producción de miel y otros productos de la colmena, el acopio de los productos, el agregado de valor por diferenciación y/o fraccionamiento y la comercialización. Es muy frecuente que un mismo actor cumpla más de una función, revelando el escaso nivel de estructuración y especialización. A su vez, la misma presenta las ventajas de ser una cadena que no pone en juego la disputa por bienes naturales escasos en el territorio (tierra y agua) $\mathrm{y}$ al interior de las propias explotaciones familiares (en los conflictos entre generaciones). Por estos motivos, se presentó como una alternativa productiva y de generación de ingresos interesante para su promoción en el marco de los programas de desarrollo rural, especialmente entre los jóvenes.

El inicio de la intervención del Prodernea en la cadena de valor apícola chaqueña se sitúa en el año 2000 en el contexto del incipiente desarrollo de la actividad provincial. Este proceso coincidió con un período en que la producción agropecuaria familiar atravesaba una profunda crisis, originada en la desfavorable estructura de precios relativos de los productos y agravada por las recurrentes inundaciones que afectaron a la provincia (CIET, 2007).

La transición experimentada en el escenario nacional a partir del año 2002 y el ajuste del tipo real de cambio produjo un incremento de la rentabilidad de los sistemas productivos agropecuarios. En este marco, la cadena de valor apícola comenzó a transitar un escenario que brindó condiciones para lograr un mayor dinamismo a partir de un ambiente propicio para la actividad apícola en la región, en función de su valor de exportación.

El fomento de esta actividad se instrumentó a través del apoyo a proyectos productivos grupales con 
302 Promoción, Desarrollo y Persistencia de la Agricultura Familiar y sus Formas Asociativas: el caso de las asociaciones apícolas en la provincia de Chaco, Argentina (1999-2015)

asistencia técnica, capacitación y financiamiento para la adquisición de colmenas y salas de extracción. La base productiva estaba conformada por un número disperso de productores, situación que reducía los espacios disponibles para la articulación de proyectos productivos grupales. La mayoría de los beneficiarios estaban comprendidos en los criterios empleados para la definición de la población objetivo del programa: productores agropecuarios familiares y empresariales que desarrollaban otras actividades de renta y de autoconsumo. Este segmento incluyó, además, a un grupo de actores sociales que desarrollaban actividades no agropecuarias y que habían adoptado la apicultura como una fuente secundaria de ingresos. El desarrollo tecnológico era marcadamente heterogéneo, con un acotado número de apicultores que exhibía altos niveles de productividad. Los rendimientos variaban de acuerdo al manejo de los apiarios y al régimen de las precipitaciones que influye sobre la floración de las especies autóctonas. En este marco, los productores familiares coexistían con otros productores que habían adoptado la actividad con una visión de negocios y que pasaron a constituirse en los impulsores del desarrollo de la cadena de valor apícola en esta provincia.

El bajo desempeño del programa en la primera fase de actuación y la necesidad de reunir a seis productores que aceptaran constituir garantías solidarias para acceder a los créditos del programa, incidieron sobre el proceso de constitución de los grupos. A esto se sumó la urgencia para realizar los primeros desembolsos, situación que originó la conformación apresurada de los grupos apícolas y la elaboración de los proyectos productivos por parte de los técnicos del programa.

De este modo, se crearon 23 grupos con un promedio de siete integrantes cada uno, cuyos resultados fueron dispares y aislados. Los técnicos a cargo de la formulación de los proyectos impusieron su percepción de la actividad apícola en los planteos inducidos a los productores, haciendo abstracción de la disponibilidad de recursos económicos de los beneficiarios, cuestión que condujo al fracaso de varios emprendimientos. La excepción a esta realidad estuvo representada por los grupos formados por técnicos que poseían experiencia laboral en otros programas de desarrollo rural o en aquellas zonas en donde las falencias de las propuestas técnicas fueron suplidas por las condiciones ambientales favorables para la producción apícola, como la presencia de una elevada población de enjambres y una abundante flora melífera.

En este escenario, algunos proyectos alcanzaron un nivel de desarrollo en donde la apicultura logró generar ingresos suficientes para realizar la devolución de los préstamos, mientras que en otros resultó evidente la imposibilidad de encarar este proceso. La información disponible muestra que el programa financió la adquisición de 6.583 colmenas (cajones vacíos y/o núcleos), de las cuales sólo el $60 \%$ lograron estar efectivamente activas (CIET, 2007).

Sibienla elevada dispersión geográfica delos grupos fue uno de los factores más importantes que limitó su integración, la debilidad de las acciones tendientes a inducir y fortalecer el desarrollo organizativo de los grupos constituyó un déficit destacable de la asistencia técnica y financiera.

La autogestión de los grupos, sobre la que debían haberse asentado las posibilidades de garantizar el cumplimiento de los objetivos del programa, no se alcanzaron. La mayoría ostentó un escaso desempeño organizacional y representaron una suma de individualidades. El programa generó capacidad de autogestión individual, aspecto que se manifestó en los conocimientos adquiridos por los productores para mantenerse en la actividad. Sin embargo, fueron pocos los que utilizaron la estructura de los grupos para vincularse con los proveedores y con los compradores de la producción.

No obstante, y en contraste con estas situaciones generales, algunos grupos pudieron permaneceractivos debido al claro liderazgo de uno de sus integrantes, otros consiguieron avanzar en la integración a la cadena de valor constituyendo una asociación (Cooperativa Apícola Chaco y Coseba) y otros ya estaban asociados a alguna cooperativa (Cooperativa Apícola Chacabuco), aunque esta situación organizacional no fue tenida en cuenta por el programa para canalizar la asistencia técnica y económica.

El programa financió la construcción y/o equipamiento de salas de extracción a través de cuatro grupos. Sin embargo, dichas salas poseían una elevada capacidad ociosa al concentrar sus operaciones en los meses de cosecha y al estar notoriamente sobredimensionadas territorialmente para la prestación de servicios a otros grupos y a terceros.

La intervención del programa en la cadena de valor apícola chaqueña se dio en un ambiente institucional 
caracterizado por reducidas instancias generadas para la articulación de las acciones en apoyo a la producción familiar. Se establecieron escasas y dificultosas acciones de colaboración con otros programas y agentes técnicos presentes en el territorio como el Programa Social Agropecuario (PSA) y el Inta. A su vez, las diferencias de enfoques y criterios entre los técnicos del programa y el Ministerio de la Producción provincial generaban una suerte de aislamiento de las acciones del programa y la agricultura familiar dentro de su órbita.

Estas cuestiones comenzaron a superarse cuando el Ministerio decidió asumir la responsabilidad de coordinar todas las intervenciones públicas que involucraban a la cadena de valor con la finalidad de potenciar el impacto de estas en el mejoramiento de su competitividad.

La institucionalidad de la cadena de valor comenzó a presentar un escenario más auspicioso al construirse en torno al Consejo Asesor Apícola Provincial, instancia integrada por representantes de los productores en cada una de las cuencas apícolas, instituciones públicas nacionales y provinciales involucradas en la cadena, referentes técnicos del Inta-Proapi (Proyecto Integrado de Desarrollo Apícola), la Universidad Nacional del Nordeste, el Servicio Nacional de Sanidad y Calidad Agroalimentaria (Senasa) y otros organismos, siendo su objeto asesorar a las autoridades en todo lo referente a la actividad apícola. En su primera fase de operación, el Consejo representaba un órgano consultivo y luego pasó a cumplir funciones resolutivas, tomando decisiones que involucraron a la cadena de valor (precios, producción y articulación organizacional). La maduración de este espacio de articulación de actores se vio reflejada posteriormente en el Plan Apícola Provincial llevado adelante por el Ministerio de la Producción con el apoyo del Prodear.

A fines de 2009, el Ministerio de la Producción definió su estrategia de intervención y presupuestos a partir de una red de programas por cadenas de valor destinadas a intervenir en sistemas productivos específicos. Inicialmente, estas cadenas fueron cinco: caprina, apícola, fruti-hortícola, foresto-ganadera y de artesanía; y a partir de 2013 se incorporaron las cadenas porcina y láctea. En el marco de esta estrategia provincial, el Prodear articuló sus acciones funcionando como una herramienta de las políticas del Ministerio bajo esta lógica, priorizando aquellas cadenas que tenían mayor relevancia para los pequeños productores familiares y articulando sus funciones técnicas y administrativas. Los técnicos financiados por el programa cumplían funciones dentro del mismo ministerio y sus informes mensuales de seguimiento eran los mismos para el programa que para el área correspondiente del Ministerio, evitando el aislamiento y la fragmentación de las intervenciones acontecidas durante la ejecución del Prodernea.

Paralelamente, se definió como segundo eje estratégico el fortalecimiento de las organizaciones de productores como un modo de generar procesos de desarrollo e inserción sostenibles de los pequeños productores en las cadenas de valor. La estrategia de intervención provincial puso especial énfasis en la integración de los productores a las cadenas, siendo las organizaciones el eje de trabajo de sus acciones.

La articulación del programa a la nueva lógica del Ministerio permitió en poco tiempo lograr una ágil ejecución de fondos gracias a las ventajas que implicó el apoyo a organizaciones ya consolidadas y a la formalización de aquellas que todavía no lo habían hecho pero que, en la práctica, funcionaban como grupos asociativos de productores, logrando de este modo ejecutar proyectos de mayor envergadura e impacto económico que los realizados en el marco del Prodernea. Teniendo en cuenta que la mayoría de los técnicos tenían un perfil agronómico o veterinario y que se veían limitados para el abordaje de las problemáticas vinculados con el desarrollo organizacional de los grupos se creó dentro del organigrama del programa un área de fortalecimiento organizacional cuyo coordinador cumplía la función de articular al equipo de gestión con los técnicos de terreno y de asistirlos en la transmisión del programa hacia las organizaciones, con la expectativa de generar procesos donde los productores asumieran mayores responsabilidades y se hicieran cargo de algunas de las múltiples tareas o roles que tendían a concentrarse en los técnicos de campo.

En mayo de 2013 el gobierno provincial chaqueño lanzó un Plan Apícola reuniendo la experiencia acumulada sobre esta cadena de valor durante los años precedentes. Para esa fecha, la provincia contaba con 16 organizaciones formales de apicultores y 2.400 productores inscriptos en el Registro Nacional de Productores Apícolas con un total de 85.000 colmenas. Contaba además con 14 salas de extracción fijas y dos móviles, una sala de fraccionamiento y dos plantas homogeneizadoras. 
304 - Promoción, Desarrollo y Persistencia de la Agricultura Familiar y sus Formas Asociativas: el caso de las asociaciones apícolas en la provincia de Chaco, Argentina (1999-2015)

Los proyectos apícolas financiados por el Prodear se articularon con dicho plan de acuerdo a las necesidades y problemas detectados en la cadena. En términos productivos, contribuyó con el soporte técnico de la actividad, el financiamiento de proyectos con fondos rotatorios administrados por las organizaciones y la reactivación y fortalecimiento de las mismas a través del componente Foco (Fondo de Capitalización de Organizaciones), así como el apoyo al Programa de Jóvenes Apicultores. ${ }^{13}$ Para la etapa de procesamiento se financiaron proyectos de readecuación de la infraestructura en salas de extracción a cargo de las organizaciones de productores con el objeto de lograr la habilitación requerida. También se financió la construcción de nuevas salas y la adquisición de equipamiento en lugares donde no estaba cubierta esta necesidad. En cuanto a la comercialización, dado que la mayoría de la producción se comercializaba a granel para exportación, se promovió el agregado de valor a partir del fraccionamiento para la participación en ferias y eventos locales donde las organizaciones apícolas pudieron ofrecer sus productos y establecer alianzas estratégicas con el sector privado.

Al 30 de junio de 2013, el Prodear-Chaco destinó el $22 \%$ de sus fondos al financiamiento de 21 proyectos apícolas correspondientes a 15 organizaciones y 735 familias. De los proyectos financiados, 14 correspondían a la línea de financiamiento (FAE) para la mejora de instalaciones, construcción de salas de extracción, compra de equipamiento y 7 a la línea del fondo de capitalización (Foco) para el fortalecimiento de la capacidad organizacional y productiva (incremento y recuperación de colmenas) de las asociaciones apícolas. ${ }^{14}$ Según informes técnicos del programa, en el período 2012-2013 se logró un crecimiento en la escala de producción del $100 \%$ y un incremento productivo del $80 \%$. Durante este período, el $90 \%$ de los productores fueron asistidos por el Ministerio de la Producción y el 65\% contó con algún financiamiento del Prodear, con la asistencia en terreno de un técnico cada 100 apicultores.

13. Este programa propone incorporar a la actividad a 200 jóvenes que cursen los últimos años de los colegios agro-técnicos y de las Escuelas de Familias Rurales (EFA) a través de cursos de capacitación en producción apícola y la posibilidad de formular proyectos que puedan ser financiados por el programa.

14. Al finalizar el programa, el número de FOCOs apícolas aumentó a 10 (FIDA, 2016).
La experiencia del Prodear en el marco de la cadena apícola chaqueña, permite observar cierta madurez de sus actores participantes, donde se han podido capitalizar las capacidades latentes en la provincia en términos de recursos naturales y humanos a partir de experiencias anteriores vinculadas a la ejecución del Prodernea. Algunos de los grupos y técnicos que perduraron en forma dispersa luego de la finalización de aquel programa, pudieron enfrentar procesos de consolidación organizacional y económica en el marco de proyectos financiados por Prodear y del Plan Apícola provincial permitiendo la creación de cooperativas formalmente constituidas, aumentar el volumen y la calidad de su producción y fortalecer su posición en el proceso de comercialización.

El caso de la Cooperativa Palmares de Basail es un ejemplo de este proceso. ${ }^{15}$ En el año 2011 se constituyó como una cooperativa agropecuaria integrada por 24 socios. Si bien su principal actividad es apícola (genera alrededor del $50 \%$ de sus ingresos), por estatuto la cooperativa se encuentra abierta a incorporar otros productos y actividades. Sus asociados son pequeños productores agropecuarios con un alto grado de diversificación en sus actividades prediales como la horticultura y la ganadería. El grupo base de la actual cooperativa proviene de la unión de dos grupos conformados entre los años 2000 y el 2002 en el marco de los proyectos del Prodernea (uno de la localidad de Basail y otro de Cote Lai) a los que se sumaron nuevos integrantes en un período posterior a la culminación de aquellos proyectos. La experiencia de participación en el Prodernea le permitió a aquel grupo de jóvenes acceder a servicios de asistencia técnica, capacitación y ayuda económica para mejorar la calidad de una actividad que, por aquel entonces, realizaban como tarea residual en las tierras de sus padres. Como jóvenes hijos de productores y dada la escasez de tierra con la que contaban, la apicultura se presentó como una actividad viable. El claro liderazgo de algunos de sus integrantes y del técnico de terreno, que siguió asistiéndolos una vez finalizado el Prodernea, les permitió fortalecer el grupo, incrementar su producción y consolidarse en la actividad.

15. Entrevista realizada en la Cooperativa donde funciona su sala de extracción en la localidad de Basail (Chaco) el 26/06/2013 con su presidente, tesorero, cinco asociados y el asesor técnico del Prodear/Ministerio de la Producción. 
Durante más de ocho años administraron en forma eficiente un fondo rotatorio para la compra de insumos. Esta estrategia les permitió autofinanciarse para persistir en el tiempo a pesar de la ausencia del Estado y del programa, financiando en pequeña escala a nuevos apicultores, comprando colmenas, mejorando la calidad de la miel producida, creciendo en volumen de producción, y vinculándose con otros apicultores para fortalecer su capacidad comercial.

En el 2013 la cooperativa contaba con 2.045 colmenas en producción con una capacidad productiva promedio de 35.000 kilos de miel anuales, las que se comercializaban a granel en tambores para exportación. Con el apoyo técnico del Prodear pudieron constituirse formalmente como cooperativa, fortaleciéndose frente a otros actores para la oferta del producto y para la compra de insumos. También construyeron una sala de extracción que se presentaba como una necesidad de la zona dado que la más cercana se encontraba a $110 \mathrm{~km}$. y porque se consideraba un "cuello de botella" problemático de su actividad productiva y comercial. El Prodear además les otorgó recursos a través del Foco con el objeto de posibilitar el financiamiento de los asociados para aumentar el volumen de producción a 160.000 kilos anuales en cinco años con un promedio de 200 colmenas por productor y también para sumar jóvenes apicultores, estudiantes de las Escuelas de Familia Agrícola (EFA) en el marco del Programa Jóvenes Apicultores.

A pesar de que la apicultura es una actividad secundaria o complementaria para la mayoría de los productores de esta cooperativa, los mismos manifestaron que con los ingresos de la apicultura realizaron reparaciones en los predios o sus maquinarias, permitiéndoles reactivar las actividades agrícolas de la chacra y ampliar sus actividades comerciales a través de acuerdos con el mercado central de la provincia para la venta hortícola de batata. Esta cuestión resulta relevante para comprender como la actividad apícola se inserta en el marco de las estrategias individuales y colectivas de producción diversificadas que son propias de los productores familiares de la zona y fundamentales para su persistencia.

En este sentido, destacan que el hecho de haber podido consolidarse como cooperativa les ha permitido vender a mejor precio, mejorar su posición frente a los demás actores de la cadena y ampliar su rama de actividades. A la vez, destacan el sistema de autofinanciación de las organizaciones propiciado por el programa ya que les dio autonomía y les permitió apropiarse de los mismos como parte de su patrimonio, afianzándolos como grupo. ${ }^{16}$

De este modo, la intervención del programa en el marco de la política provincial de apoyo a la apicultura, contrasta notoriamente con la precedente del Prodernea en esta materia. De focalizar en pequeños grupos creados ad hoc de 5 a 7 productores, se pasó a un apoyo a las organizaciones ya consolidadas o en proceso de consolidación con la consigna de que éstas incorporen a los productores dispersos. Además de una intervención acotada a lo productivo, se pasó a una visión integral de la cadena donde se privilegiaron los eslabones vinculados con el procesamiento y la comercialización como forma de elevar los ingresos y la productividad. Por último, la estrategia donde los créditos y subsidios eran administrados por el programa y la provincia a través de sus técnicos, fue desplazada por un procedimiento donde los fondos rotatorios son administrados por los beneficiarios a partir de sus organizaciones, las cuales a su vez crecen y se consolidan en dicho proceso con el asesoramiento de los técnicos.

Estas cuestiones, lejos están de presentarse como formulas o modelos ideales a seguir, ya que también presentaron dificultades o no tuvieron el mismo "éxito" en el marco de otras cadenas de la misma provincia, como la caprina, donde la mayoría de los grupos trabajaron en forma conjunta en la práctica, pero les costó trascender y sostener organizaciones con algún grado de formalidad y persistencia en el tiempo. No obstante, aún en el marco de estas experiencias menos "exitosas", la mayoría de los destinatarios destacaron los problemas derivados de la comercialización como una situación que los pequeños productores no pueden resolver solos sin articular acciones en forma colectiva y sin la presencia del Estado como mediador en los procesos de inserción de sus productos en el mercado frente a otros actores de mayor peso económico y político. Esto se ve claramente reflejado en la relatoría de los talleres de evaluación final del PRODEAR, donde sus destinatarios hicieron una valoración positiva de

16. En algunos de los documentos de finalización del programa (UCAR-PRODEAR, 2015; FIDA-PRODEAR, 2015), se pueden observar más experiencias apícolas y de otros rubros que arriban a conclusiones similares y que por cuestiones de espacio no transcribimos en este artículo. 
306 - Promoción, Desarrollo y Persistencia de la Agricultura Familiar y sus Formas Asociativas: el caso de las asociaciones apícolas en la provincia de Chaco, Argentina (1999-2015)

las experiencias asociativas desarrolladas más allá del grado de beneficios económicos que éstas les hayan posibilitado (UCAR-PRODEAR, 2015).

\section{Discusión}

\subsection{Cinco ejes de reflexión a partir del estudio de caso propuesto}

Del análisis propuesto a lo largo de este escrito se desprenden cinco ejes de reflexión para la discusión en torno a las posibilidades de desarrollo y persistencia de la agricultura familiar en los territorios rurales y sus estrategias de promoción en el marco de las políticas públicas. Los mismos nos permiten trazar algunas líneas teórico-prácticas de contrastación con otros casos y futuras líneas indagatorias.

El primer eje de reflexión refiere al indelegable e imprescindible rol del Estado como promotor, mediador y regulador en los procesos de desarrollo y del diálogo político entre los actores intervinientes en el marco de una cadena productiva y los territorios rurales. La experiencia apícola analizada demuestra el rol central que ha cumplido el Estado en sus diferentes niveles para la inclusión de los pequeños productores familiares en la cadena y como concertador entre la variedad de agentes institucionales e interinstitucionales públicoprivado intervinientes en el marco de acciones que combinan lo urbano con lo rural. Una vez finalizados los programas y sin caer en los vicios del clientelismo (técnico o político), el Estado no puede pretender desentenderse de los pequeños productores familiares en tanto referencia económica, simbólica y política sobre la que descansa buena parte de sus posibilidades de reproducción en el agro moderno.

El segundo eje de reflexión a partir del caso estudiado, alude a la importancia que han tenido las estrategias de desarrollo tendientes al fortalecimiento de las organizaciones y grupos de productores para que sean capaces de autogestionarse y participar de actividades comerciales, así como convertirse en interlocutores del diálogo político con actores de otros estratos y con los agentes del Estado. En la experiencia del Prodear, las acciones de fortalecimiento organizacional de los productores han sido tan importantes como las acciones para el mejoramiento de sus capacidades productivas y de acceso a los mercados, demostrando la importancia del fortalecimiento de las organizaciones locales y acciones de capacitación para la autogestión que garantizaron la sostenibilidad de las acciones encaminadas por los proyectos. El caso apícola presentado, demuestra como una cadena residual en términos productivos y de ingresos para los productores de la zona y con escaso grado de complejidad, sirvió de escusa para organizarse y, a partir de eso, pudieron comenzar a elaborar acciones cooperativas en otros rubros potenciando el carácter diversificado de su producción.

El tercer eje, estrechamente vinculado al anterior, refiere al papel desempeñado por los técnicos extensionistas de los programas de desarrollo rural. La experiencia analizada, junto a otras acciones vinculadas al desarrollo organizacional de la agricultura familiar en el mismo período (IURMAN, 2016), demuestran el rol fundamental que han cumplido como agentes mediadores entre el Estado y los productores del medio rural, desbordando ampliamente sus funciones técnico-productivas. En ambos programas los técnicos sugirieron a los equipos de gestión cuáles eran los grupos u organizaciones de productores que estaban en condiciones de sostener un proyecto que implicara asumir crédito o elaborar planes para la administración de aportes no reembolsables para su autofinanciamiento. Durante la ejecución del Prodernea, los técnicos quedaron como los referentes y responsables de los proyectos más que los productores y sus representantes. Cuestión que intentó ser remediada en el marco de las acciones del Prodear, aunque igualmente, por el grado de madurez organizacional que implicó la administración de fondos y proyectos productivos y comerciales, requirieron de la dedicación especializada de los técnicos y cierto protagonismo de los mismos como asesores. También, cabe agregar que, tal como sostiene Schiavoni (2012), fueron los técnicos los que han desempeñado el papel de "traductores" del término agricultura familiar en el marco de los programas de desarrollo, conectando seres que de otro modo permanecerían aislados y desprovistos de significado. Fueron los técnicos quienes, basándose en lazos personalizados con los sujetos en los territorios, han definido el perfil de los actores beneficiarios de los programas que se han constituido en el referente empírico de la categoría. En este sentido, hay que resaltar el papel central desempeñado por los técnicos de los programas de 
desarrollo rural en la promoción de la agricultura familiar y la conformación de sus asociaciones económicas y reivindicativas en Argentina.

El cuarto eje y siguiendo encadenadamente estas reflexiones, hace alusión a un aspecto que es intrínseco a la noción de capital social desde las diferentes perspectivas teóricas que la promueven. Nos referimos al hecho de que su adquisición y desarrollo necesitan de tiempo (trayectoria), dedicación (inversión de tiempo por parte de personas) y continuidad (acciones sostenidas en el tiempo). Los teóricos del capital social comparten una visión dinámica del mismo, en tanto conjunto de recursos (BOURDIEU, 1980) o beneficios derivados de actos de intercambio recíproco -redes, confianza, normas, etc.- (COLEMAN, 2000; PUTNAM, 1993) que pueden ser creados, mantenidos o destruidos. Por ello, es importante destacar que la experiencia asociativa promovida por los programas, como un capital social no puede ser implantada de un día para otro y requiere de acciones continuas y sostenidas en el tiempo por parte de los actores y las agencias del Estado. Si bien los resultados asociativos del Prodernea no fueron los deseados en función de la persistencia y grado de acción colaborativa de los grupos creados, generó una serie de procesos relevantes y no explícitamente planificados como la formación de líderes y dirigentes para la representatividad sectorial de la agricultura familiar y de personal técnico para la generación y gestión de instrumentos de política pública que luego fueron capitalizados en el marco del Prodear y otras experiencias asociativas como el FoNAF. En la misma dirección, la trayectoria de la Cooperativa Palmares de Basail resume con claridad la importancia del tiempo transcurrido en la consolidación del capital social, en tanto sus integrantes pasaron de ser un grupo informal de jóvenes apicultores en el Prodernea, a una organización de productores apícolas (adultos) con alto grado de maduración en el Prodear. La capitalización de las experiencias previas de estos agentes fueron las que permitieron encaminar exitosamente sus proyectos y también la continuidad de sus acciones una vez finalizados los programas.

Este aspecto nos lleva a un último eje de reflexión en torno a estas formas asociativas integradas por los sujetos que en sentido amplio comprendemos como agricultores familiares. La experiencia del Prodernea nos demuestra que, paradójicamente, los grupos basados en la extensión del lazo doméstico (familiar) que caracterizaron a las asociaciones de productores de aquel programa y que reforzaban la idea de una agricultura familiar en el territorio, no garantizaron por sí solos la persistencia y consolidación de acciones cooperativas. Por ello, cuando nos referimos a las formas asociativas de la agricultura familiar no estamos haciendo estricta referencia a las condiciones prediales o filiares de quienes las integran, sino más bien al carácter colectivo de las acciones económicas y políticas de los actores rurales que, por su inserción territorial y local, se identifican con la agricultura familiar como práctica socio-productiva y estilo de vida específico. Teniendo en cuenta que la agricultura familiar no es una categoría estática que permanece fija, sino que trata de representar la realidad de grupos en procesos de transición "hacia" o "resistiendo la transición hacia" (MURMIS, 1994) y que el eje agrario de la estrategia familiar de reproducción social, puede cambiar de acuerdo a las circunstancias del contexto social, lo central no parece ser la familia ni la agricultura, sino el modo en que los diferentes actores se insertan territorial y localmente en el medio rural, puesto que, en definitiva, es el territorio el lugar de reproducción de lo rural como "modo de vida" y no la sola existencia de mano de obra familiar en los predios. Es decir, el modo en que los habitantes del territorio (campesinos, comunidades aborígenes, familias agricultoras "modernas", trabajadores y pobladores rurales) se organizan y articulan acciones para decidir sobre los usos sociales y productivos del espacio con cierto grado de empoderamiento respecto de otros actores del agro moderno con mayor capacidad de negociación y representación política y económica (agricultores empresariales, complejos agroindustrias, proveedores de insumos, agentes de comercialización, etc.).

De este modo, parece pertinente propiciar estrategias de desarrollo en los territorios rurales que busquen el fortalecimiento de la agricultura familiar y sus organizaciones, no sólo como una alternativa económica para enfrentar las debilidades de los productores frente al mercado, sino también para generar reconocimiento (visibilidad) y mayor capacidad de participación en los procesos de interlocución con el Estado para la definición de las políticas públicas. 
308 - Promoción, Desarrollo y Persistencia de la Agricultura Familiar y sus Formas Asociativas: el caso de las asociaciones apícolas en la provincia de Chaco, Argentina (1999-2015)

\section{Conclusiones}

El sector agropecuario argentino se expresa en la actualidad en una densa red asociativa construida a partir de diversas formas organizativas y tramas de articulación público-privadas, en las que participan productores agropecuarios y población rural de diferentes condiciones y disponibilidad de recursos económicos y productivos. Esta red asociativa se ha ido construyendo desde los inicios de la agricultura en la Argentina y en buena medida han sido producto tanto del dinamismo de las fuerzas productivas y sociales en el agro, como de las políticas públicas.

La retracción del Estado en la década de 1990 y los cambios estructurales en la economía, contribuyeron a una intensificación del pluralismo asociativo en el agro, con el surgimiento de cientos de nuevas asociaciones o proyectos asociativos, en algunos casos autogenerados por los actores socioeconómicos en respuesta al cambio de las reglas de juego, y otros promovidos activamente por los programas de desarrollo rural focalizados, tanto públicos como privados, que incorporaron la estrategia asociativa como base de su intervención en favor de los estratos medios y bajos de la estructura agraria.

Si bien la agricultura familiar no había sido una cuestión relevante de la agenda sectorial, la experiencia desarrollada durante la década de 1990 sirvió para la creación de un tejido conectivo entre productores, técnicos, organizaciones y agencias estatales que fortalecieron progresivamente su visibilidad y acción colectiva. De este modo, en la primera década del siglo XXI, su problemática fue creciendo en importancia en la región y en el ámbito nacional a partir de una estrategia gubernamental explícita en la reorientación de los programas de desarrollo rural con el objeto de fortalecer la organización y representación de intereses de estos sectores rurales.

En este artículo nos hemos propuesto analizar ese cumulo de experiencias asociativas promovidas en la continuidad de las intervenciones de dos programas de desarrollo rural en la provincia de Chaco, prestando especial atención al caso de los grupos asociativos apícolas.

El tratamiento de esta temática en el marco de dichos programas se constituyó en una excelente plataforma de observación para dar cuenta de distintos momentos de contextualización de la agricultura familiar y el asociativismo como estrategia de intervención.
Sus primeras experiencias en el marco del Prodernea tomaban como población objetivo a los pequeños productores en condiciones de pobreza de las provincias del NEA a los que, a partir de 2003 y luego de un cambio significativo de su funcionamiento y enfoques, comenzaron a identificar como agricultores familiares; redefiniendo al mismo tiempo sus estrategias de intervención en el marco del Prodear, donde se comienza a dar mayor centralidad al fortalecimiento de las organizaciones del sector, en sintonía con las propuestas del Desarrollo Territorial Rural y del capital social.

El caso concreto de los proyectos apícolas, nos permitió señalar las posibilidades de persistencia y desarrollo organizacional de los grupos a través de los años de gestión de estos programas. Es decir, nos permitió señalar, tanto aspectos instrumentales del capital social (en tanto "herramienta para la intervención y el desarrollo"), como aspectos analíticos intrínsecos a los valores, normas, actitudes y redes de relaciones existentes entre las personas y organizaciones de una comunidad (en tanto factores claves para la comprensión de los procesos de desarrollo y la consolidación de la agricultura familiar como actor político y económico).

Teniendo en cuenta estos aspectos teóricos y empíricos, hemos indicado, entonces, el proceso de jerarquización institucional de la agricultura familiar en el ámbito de las políticas públicas con énfasis en el desarrollo de los territorios rurales, en paralelo con un incremento de las acciones colectivas de los actores que integran su base social, aunque con cierto anclaje de dependencia con los cuadros técnicos y políticos que promovieron su conformación. Este proceso se encuentra operando actualmente en los territorios rurales y plantean numerosos interrogantes sobre su dimensión, característica y potencialidad que aún no están cerrados y que deben cotejarse con el nuevo contexto económico, político e institucional que se inicia en Argentina a finales del 2015.

\section{Referencias}

BAUDRÓN, S. Programa de crédito y apoyo técnico para pequeños productores del noroeste argentino. En: FLORES, G. (Comp.). De agricultor a pequeño empresario con crédito agrícola. Experiencias y procesos futuros. Santiago de Chile: PROCEDER, INDAP, IICA, 1993, p. 77-84. 
BOURDIEU, P. Le capital social. Notes provisoires. Actes de la Recherche en Sciencies Sociales, n. 3, p. 2-3, 1980.

CEDES - Centro de Estudios de Estado y Sociedad. Diagnóstico sobre la contribución del PRODERNEA al capital social de los actores, sus organizaciones y comunidades. Buenos Aires: CEDES, 2007.

CIET-Centro Interdisciplinario de Estudio Territoriales. Sistematización de la experiencia e intervención del PRODERNEA en la producción apícola en la Provincia de Chaco. Resistencia, Argentina: CIET, 2007.

COLEMAN, J. Social capital in the creation of human capital. Social capital. A multi-faceted perspective. Washington: World Bank, 2000.

CRAVIOTTI, C. La agricultura familiar en Argentina: nuevos desarrollos institucionales, viejas tendencias estructurales. En: . (Comp.). Agricultura familiar en Lationamérica: continuidades, transformaciones y controversias. Buenos Aires: Ciccus, 2014, p. 175-204.

CUÉLLAR SAAVEDRA, O. y BOLIVAR ESPINOZA, G. A. Capital social hoy. Polis, v. 8, n. 22, p. 195-217, 2009.

DURSTON, J. El capital social en seis comunidades campesinas de Chile: adelantos y desafíos de una investigación en marcha. En: DURSTON, J. y MIRANDA, F. (Comps.). Capital social y políticas públicas en Chile. Investigaciones recientes. Santiago de Chile: CEPAL, 2001, p. 39-65.

FIDA - Fondo Internacional para el Desarrollo Agrícola. Proyecto de Desarrollo Rural de las Provincias del Noroeste de Argentina (PRODERNEA). Informe Misión de Reorientación. Buenos Aires: FIDA, 2002.

Proyecto de Desarrollo Rural de las Provincias

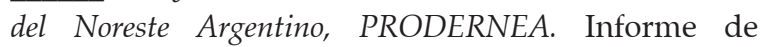
Terminación del Proyecto. Buenos Aires: FIDA, 2008.

PRODERI (713AR) y PRODEAR (848-AR y

$\overline{E-4-A R}$ ). Informe de supervisión. Buenos Aires: Fondo Internacional para el Desarrollo Agrícola (FIDA), 2016.

FIDA-PRODEAR - Fondo Internacional para el Desarrollo Agrícola - Programa de Desarrollo de Áreas Rurales. Riqueza latente IV. Otra mirada sobre el desarrollo rural. Buenos Aires: FIDA-PRODEARUCAR-MAGyP, 2015.

FLEXOR, G. y GRISA. Institutionalization of family farm policy in Brazil: history, ideas and actors. En: $1^{\text {st }}$ INTERNATIONAL CONFERENCE ON PUBLIC POLICY. Grenoble, France: University of Grenoble, 2013. Disponible en: < http://www.icpublicpolicy.org / IMG/pdf/panel_26_s1_flexor_grisa.pdf > . Acceso en: 13 nov. 2013.
GARCÍA OJEDA, M. Capital social y clientelismo: otra limitación para el control social. Polis [en línea], n. 29, 2011. Disponible en: <http://polis.revues.or/1943>. Acceso en: 20 de mayo de 2016.

IURMAN, J. P. Algunas transformaciones en los dispositivos de desarrollo rural, en los roles de los mediadores y en las organizaciones de la agricultura familiar a partir de la creación de la Subsecretaría de Agricultura Familiar en la República Argentina. El caso de la Federación de Organizaciones Nucleadas de la Agricultura Familiar. En: PRE CONGRESO ALASRU, LA SOCIOLOGÍA EN LA ENCRUCIJADA: VIGENCIA DE LA CUESTIÓN AGRARIA, ACTORES SOCIALES Y MODELOS DE DESARROLLO EN LA REGIÓN. Santiago del Estero, Argentina: Universidad Nacional de Santiago del Estero, 2016.

LATTUADA, M. Cambio rural. Política y desarrollo en la argentina de los `90. Buenos Aires: CED-Acasur, 2000.

LATTUADA, M., NOGUEIRA, M. E. y URCOLA, M. Las formas asociativas de la agricultura familiar en el desarrollo rural argentino de las últimas décadas (19902014). CIRIEC-España, Revista de Economía Pública, Social y Cooperativa, n. 84, p. 195-228, 2015a.

. Tres décadas de desarrollo rural en la Argentina. Continuidades y rupturas de intervenciones públicas en contextos cambiantes (1984-2014). Buenos Aires: Teseo-UAI, 2015b.

LIN, N. Social capital: theory of social structure and action. Cambridge: Cambridge University Press, 2001.

MAGyP - Ministerio de Agricultura, Ganadería y Pesca de la Nación. Programa de Desarrollo de Áreas Rurales (PRODEAR). Manual Operativo. Buenos Aires: MAGyP, 2010.

MURMIS, M. Algunos temas para la discusión en la Sociología Rural Latinoamericana: reestructuración, desestructuración y problemas de excluidos e incluidos. Ruralia, Revista Argentina de Estudios Agrarios de FLACSO, n. 5, p. 43-68, 1994.

NEIMAN, G. y QUARANTA, G. Los estudios de caso en la investigación sociológica. En: VASILACHIS DE GIALDINO, I. (Coord.). Estrategias de investigación cualitativa. Barcelona: Gedisa, 2006, p. 213-237.

et al. Diversidad de las formas de representación de intereses entre organizaciones de pequeños productores del agro argentino: base social, reivindicaciones y articulaciones. En: MANZANAL, M., NEIMAN, G. y LATTUADA, M. (Comps.). Desarrollo rural. Organizaciones, instituciones y territorios. Buenos Aires: Ciccus, 2006, p. 177-210. 
310 - Promoción, Desarrollo y Persistencia de la Agricultura Familiar y sus Formas Asociativas: el caso de las asociaciones apícolas en la provincia de Chaco, Argentina (1999-2015)

NOGUEIRA, M. E. y URCOLA, M. La jerarquización de la agricultura familiar en las políticas de desarrollo rural en Argentina y Brasil (1990-2011). Revista IDeAS. Interfaces em Desenvolvimento, Agricultura e Sociedade, v. 7 , n. 2 , p. $96-137,2013$.

- La agricultura familiar en el marco de los programas de desarrollo rural del FIDA en el norte argentino (1991-2014). Ager. Revista de Estudios sobre Despoblación y Desarrollo Rural, n. 19, p. 7-44, 2015.

NORTH, D. Instituciones, cambio institucional y desempeño económico. México: Fondo de Cultura Económica, 1995.

PROINDER - Proyecto de Desarrollo de Pequeños Productores Agropecuarios. Los programas de desarrollo rural ejecutados en el ámbito de la SAGPyA. Buenos Aires: PROINDER, SAGPyA, Ministerio de Economía y Producción, 2003.

PUTNAM, R. Making Democracy Work. Civic traditions in mordern Italy. Princeton: Princeton University Press, 1993.

RÍOS CÁSZARES, A. y RÍOS FIGUEROA, J. Capital social y democracia: una revisión crítica de Robert Putnam. Política y Gobierno, v. VI, n. 2, p. 513-528, 1999.

SCHEJTMAN, A. y BARSKY, O. (Comps.). El desarrollo rural en la argentina. Un enfoque territorial. Buenos Aires: Siglo XXI, 2008.

y RAMÍREZ, E. Desarrollo territorial rural. Aspectos destacados de experiencias en proceso en América Latina. Valparaíso: Grupo Chorlaví, 2004.
SCHIAVONI, G. Organizaciones agrarias y constitución de categorías sociales. Plantadores y campesinos en el nordeste de Misiones (Arg.). Estudios Regionales, v. 10, n. 20, p. 7-21, 2001.

- Objetivación y medida: el registro de la agricultura familiar en Misiones. En: MANZANAL, M. y PONCE, M. (Coords.). La desigualdad idel desarrollo?: controversias del desarrollo rural en el Norte Argentino. Buenos Aires: Ciccus, 2012, p. 51-68.

SCHNEIDER, S. La agricultura familiar en América Latina. Un nuevo análisis comparativo. Roma: Fondo Internacional de Desarrollo Agrícola (FIDA), 2014.

UCAR-PRODEAR - Unidad para el Cambio Rural - Programa de Desarrollo de Áreas Rurales. Informe de talleres de evaluación de la provincia de Chaco. Relatoría/Sistematización de Talleres de Evaluación y Lineamientos Estratégicos. Buenos Aires: UCARPRODEAR, 2015.

WOOLCOCK, M. Social capital and economic development. Toward a theoretical synthesis and policy framework. Theory and Society, n. 27, p. 151-208, 1998.

WORLD BANK. The initiative on defining, monitoring and measuring social capital. Social Capital Initiative, Woking papers № 2. Washington: World Bank, 1998.

YIN, R. y HELAD, K. Using the case survey method to analyse policy studies. Administrative Science Quarterly, n. 20 , p. $371-381,1975$.

Todo o conteúdo deste periódico, exceto onde estiver identificado, está licenciado sob uma Licença Creative Commons (cc by 4.0) 